\title{
New periodic solutions for a class of singular Hamiltonian systems
}

Xiong-rui Wang ${ }^{1 *}$ and Shengyi Shen ${ }^{2}$

${ }^{\text {*Correspondence: }}$
wxr888x@163.com
${ }^{1}$ Department of Mathematics, Yibin
University, Yibin, Sichuan 644007,
China
Full list of author information is
available at the end of the article

available at the end of the article

\begin{abstract}
We use the variational minimizing method to study the existence of new nontrivial periodic solutions with a prescribed energy for second order Hamiltonian systems with singular potential $V \in C^{1}\left(R^{n} \backslash\{0\}, R\right)$, which may have an unbounded potential well.
\end{abstract}

MSC: $34 \mathrm{C} 15 ; 34 \mathrm{C} 25 ; 58 \mathrm{~F}$

Keywords: singular Hamiltonian systems; periodic solutions; variational methods

\section{Introduction and main results}

For singular Hamiltonian systems with a fixed energy $h \in R$,

$$
\begin{aligned}
& \ddot{q}+V^{\prime}(q)=0, \\
& \frac{1}{2}|\dot{q}|^{2}+V(q)=h .
\end{aligned}
$$

Ambrosetti-Coti Zelati $[1,2]$ used Ljusternik-Schnirelmann theory on an $C^{1}$ manifold to get the following theorem.

Theorem 1.1 (Ambrosetti-Coti Zelati [1]) Suppose $V \in C^{2}\left(R^{n} \backslash\{0\}, R\right)$ satisfies

(A0)

$$
V(u) \rightarrow-\infty, \quad u \rightarrow 0,
$$

(A1)

$$
3 V^{\prime}(u) \cdot u+\left(V^{\prime \prime}(u) u, u\right) \neq 0
$$

$$
V^{\prime}(u) \cdot u>0, \quad u \neq 0
$$

(A3) $\exists \alpha>2$, s.t.

$$
V^{\prime}(u) \cdot u \leq-\alpha V(u),
$$


(A4) $\exists \beta>2, r>0$, s.t.

$$
V^{\prime}(u) \cdot u \geq-\beta V(u), \quad 0<|u|<r
$$

(A5)

$$
\limsup _{|u| \rightarrow+\infty}\left[V(u)+\frac{1}{2} V^{\prime}(u) u\right] \leq 0 .
$$

Then (1.1)-(1.2) have at least one non-constant periodic solution.

After Ambrosetti-Coti Zelati, a lot of mathematicians studied singular Hamiltonian systems. Here we only mention a related recent paper of Carminati-Sere-Tanaka [3], in which they used complex variational and geometrical and topological methods to generalize Pisani's results [5]. They got the following theorems.

Theorem 1.2 Suppose $h>0, L_{0}>0$ and $V \in C^{\infty}\left(R^{n} \backslash\{0\}, R\right)$ satisfies (A0), (A4), and

(B1) $V(q) \leq 0$;

(B2) $V(q)+\frac{1}{2} V^{\prime}(q) q \leq h, \forall|q| \geq e^{L_{0}}$;

(B3) $V(q)+\frac{1}{2} V^{\prime}(q) q \geq h, \forall|q| \leq e^{-L_{0}}$.

Then (1.1)-(1.2) have at least one periodic solution with the given energy $h$ and whose action is at most $2 \pi r_{0}$ with

$$
r_{0}=\max \left\{[2(h-V(q))]^{\frac{1}{2}} ;|q|=1\right\} .
$$

Theorem 1.3 Suppose $h>0, \rho_{0}>0$ and $V \in C^{\infty}\left(R^{n} \backslash\{0\}, R\right)$ satisfies (B1), (A4), and

(B2') $\lim _{|q| \rightarrow+\infty} V^{\prime}(q)=0$;

(B3') $V(q)+\frac{1}{2} V^{\prime}(q) q \geq h, \forall|q| \leq \rho_{0}$.

Then (1.1)-(1.2) have at least one periodic solution with the given energy $h$ and whose action is at most $2 \pi r_{0}$.

Using variational minimizing methods, we get the following theorem.

Theorem 1.4 Suppose $V \in C^{1}\left(R^{n} \backslash\{0\}, R\right)$ satisfies

(V1) $\exists \alpha>0, \beta>2, r>0$, s.t.

$$
V(q) \leq-\alpha|q|^{-\beta}, \quad 0<|q|<r
$$

$$
V(q)<0, \quad q \neq 0
$$

$$
V(-q)=V(q), \quad q \neq 0 .
$$

Then for any $h>0,(1.1)-(1.2)$ have at least one non-constant periodic solution with the given energy $h$. 


\section{A few lemmas}

Let

$$
H^{1}=W^{1,2}\left(R / Z, R^{n}\right)=\left\{u: R \rightarrow R^{n}, u \in L^{2}, \dot{u} \in L^{2}, u(t+1)=u(t)\right\} .
$$

Then the standard $H^{1}$ norm is equivalent to

$$
\|u\|=\|u\|_{H^{1}}=\left(\int_{0}^{1}|\dot{u}|^{2} d t\right)^{1 / 2}+\left|\int_{0}^{1} u(t) d t\right|
$$

Let

$$
\Lambda=\left\{u \in H^{1} \mid u(t) \neq 0, \forall t\right\}
$$

By symmetry condition (V3), similar to Ambrosetti-Coti Zelati [1], let

$$
\Lambda_{0}=\left\{u \in H^{1}=W^{1,2}\left(R / Z, R^{n}\right), u(t+1 / 2)=-u(t), u(t) \neq 0\right\} .
$$

We define the equivalent norm in $E=\left\{u \in H^{1}=W^{1,2}\left(R / Z, R^{n}\right), u\left(t+\frac{1}{2}\right)=-u(t)\right\}$ :

$$
\|u\|=\|u\|_{E}=\left(\int_{0}^{1}|\dot{u}|^{2} d t\right)^{1 / 2}
$$

Lemma 2.1 $([1,4])$ Let $f(u)=\frac{1}{2} \int_{0}^{1}|\dot{u}|^{2} d t \int_{0}^{1}(h-V(u)) d t$ and $\tilde{u} \in \Lambda$ be such that $f^{\prime}(\tilde{u})=0$ and $f(\tilde{u})>0$. Set

$$
\frac{1}{T^{2}}=\frac{\int_{0}^{1}(h-V(\tilde{u})) d t}{\frac{1}{2} \int_{0}^{1}|\dot{\tilde{u}}|^{2} d t} .
$$

Then $\tilde{q}(t)=\tilde{u}(t / T)$ is a non-constant T-periodic solution for (1.1)-(1.2). Furthermore, if $V(x)<h, \forall x \neq 0$, then $f(u) \geq 0$ on $\Lambda$ and $f(u)=0, \forall u \in \Lambda$ if and only if $u$ is a nonzero constant.

If $\tilde{u} \in \Lambda_{0}$ such that $f^{\prime}(\tilde{u})=0$ and $f(\tilde{u})>0$, then we find that $\tilde{q}(t)=\tilde{u}(t / T)$ is a non-constant T-periodic solution for (1.1)-(1.2).

Lemma 2.2 (Gordon [6]) Let V satisfy the so-called Gordon Strong Force condition: There exist a neighborhood $\mathcal{N}$ of 0 and a function $U \in C^{1}(\Omega, \mathbb{R})$ such that:

(i) $\lim _{s \rightarrow 0} U(x)=-\infty$;

(ii) $-V(x) \geq\left|U^{\prime}(x)\right|^{2}$ for every $x \in \mathcal{N}-\{0\}$

Let

$$
\partial \Lambda=\left\{u \in H^{1}=W^{1,2}\left(R / Z, R^{n}\right), \exists t_{0}, u\left(t_{0}\right)=0\right\} .
$$

Then we have

$$
\int_{0}^{1} V(u) d t \rightarrow-\infty, \quad \forall u_{n} \rightarrow u \in \partial \Lambda .
$$


Let

$$
\partial \Lambda_{0}=\left\{u \in H^{1}=W^{1,2}\left(R / Z, R^{n}\right), u\left(t+\frac{1}{2}\right)=-u(t), \exists t_{0}, u\left(t_{0}\right)=0\right\} .
$$

Then we have

$$
\int_{0}^{1} V(u) d t \rightarrow-\infty, \quad \forall u_{n} \rightarrow u \in \partial \Lambda_{0} .
$$

Lemma 2.3 Let $X$ be a Banach space, and let $E \subset X$ be a weakly closed subset. Suppose that $\phi(u)$ is defined on an open subset $\Lambda \subset X$ and $\phi(u) \neq-\infty$ for any $u \in \Lambda$. Let $\phi(u)=+\infty$ for $u \in \partial \Lambda$. Assume $\phi(u) \not \equiv+\infty$ and is weakly lower semi-continuous on $\bar{\Lambda} \cap E$, and that it is coercive on $\Lambda \cap E$ :

$$
\phi(u) \rightarrow+\infty, \quad\|u\| \rightarrow+\infty
$$

and

$$
\phi\left(u_{n}\right) \rightarrow+\infty, \quad u_{n} \rightarrow u \in \partial \Lambda .
$$

Then $\phi$ attains its infimum in $\Lambda \cap E$.

Proof We set

$$
c=\inf _{\Lambda \cap E} \phi(u) .
$$

Then

$$
-\infty<c<+\infty \text {, }
$$

in fact, by the assumptions, it is obvious that $c<+\infty$. Now if $c=-\infty$, then there exists $\left\{u_{n}\right\} \subset \Lambda \cap E$ such that $\phi\left(u_{n}\right) \rightarrow-\infty$. Then we know that $\left\{u_{n}\right\}$ is bounded, since $\phi$ is coercive. By the Eberlein-Schmulyan theorem, $\left\{u_{n}\right\}$ has a weakly convergent subsequence. Finally, by the definition for $c$ and the assumption for the weakly lower semi-continuity for $\phi(u)$, we know $\phi(u)=-\infty$. This is a contradiction.

Now we know that there exists minimizing sequence $\left\{u_{n}\right\}$ such that $\phi\left(u_{n}\right) \rightarrow c$. Furthermore by the coercivity of $\phi$ we know that $\left\{u_{n}\right\}$ is bounded; then $\left\{u_{n}\right\}$ has a weakly convergent subsequence. We claim the weak limit $u \in \Lambda$, since otherwise $\phi(u)=+\infty$ by the assumption. On the other hand, by the definition of the infimum $c$ and the assumption for the weak lower semi-continuity for $\phi(u)$ on $\bar{\Lambda} \cap E$, we know $\phi(u)=c<+\infty$. This is a contradiction. So the weak limit $u \in \Lambda \cap E$ and $\phi(u)=c$.

\section{The proof of Theorem 1.4}

Lemma 3.1 Assume (V1) hold, then for any weakly convergent sequence $u_{n} \rightarrow u \in \partial \Lambda_{0}$, we have

$$
f\left(u_{n}\right) \rightarrow+\infty
$$


Proof Notice that (V1) imply Gordon's strong force condition. By the weak limit $u \in \partial \Lambda$ and $V$ satisfying Gordon's strong force condition, we have

$$
\int_{0}^{1}-V\left(u_{n}\right) d t \rightarrow+\infty, \quad \forall u_{n} \rightarrow u \in \partial \Lambda .
$$

By $u_{n} \rightarrow u$ in the Hilbert space $H^{1}$, we know that $\left\|u_{n}\right\|$ is bounded.

(1) If $u \equiv 0$, then by Sobolev's embedding theorem, we have the uniform convergence property:

$$
\left|u_{n}\right|_{\infty} \rightarrow 0, \quad n \rightarrow+\infty
$$

By the symmetry of $u(t+1 / 2)=-u(t)$, we have $\int_{0}^{1} u(t) d t=0$, then we have Sobolev's inequality:

$$
\int_{0}^{1}|\dot{u}(t)|^{2} d t \geq 12|u(t)|_{\infty}^{2}
$$

Then we have

$$
f\left(u_{n}\right) \geq 6\left|u_{n}\right|_{\infty}^{2-\beta} \rightarrow+\infty, \quad n \rightarrow+\infty
$$

So in this case we have

$$
\liminf f\left(u_{n}\right)=+\infty \geq f(u)
$$

(2) If $u \neq 0$, then we have the following. By the weakly lower semi-continuity for the norm, we have

$$
\liminf \left\|u_{n}\right\| \geq\|u\|>0
$$

So, by Gordon's lemma, we have

$$
\begin{aligned}
\liminf f\left(u_{n}\right) & =\liminf \left(\frac{1}{2} \int_{0}^{1}\left|\dot{u}_{n}\right|^{2} d t\right) \int_{0}^{1}\left(h-V\left(u_{n}\right)\right) d t=+\infty \\
& \geq \frac{1}{2} \int_{0}^{1}|\dot{u}|^{2} d t \int_{0}^{1}(h-V(u)) d t=f(u) .
\end{aligned}
$$

Lemma $3.2 f(u)$ is weakly lower semi-continuous on $\bar{\Lambda}_{0}$.

Proof For any $\left\{u_{n}\right\} \subset \bar{\Lambda}_{0}: u_{n} \rightarrow u$, by Sobolev's embedding theorem, we have uniform convergence:

$$
\left|u_{n}(t)-u(t)\right|_{\infty} \rightarrow 0
$$

(i) If $u \in \Lambda_{0}$, then by $V \in C^{1}\left(R^{n} \backslash\{0\}, R\right)$, we have

$$
\left|V\left(u_{n}(t)\right)-V(u(t))\right|_{\infty} \rightarrow 0
$$


By the weakly lower semi-continuity for norm, we have

$$
\liminf \left\|u_{n}\right\| \geq\|u\|
$$

Hence

$$
\begin{aligned}
\liminf f\left(u_{n}\right) & =\liminf \left(\frac{1}{2} \int_{0}^{1}\left|\dot{u}_{n}\right|^{2} d t\right) \int_{0}^{1}\left(h-V\left(u_{n}\right)\right) d t \\
& \geq \frac{1}{2} \int_{0}^{1}|\dot{u}|^{2} d t \int_{0}^{1}(h-V(u)) d t=f(u) .
\end{aligned}
$$

(ii) If $u \in \partial \Lambda_{0}$, then by $\Lambda$ satisfying Gordon's strong force condition, we have

$$
\int_{0}^{1}-V\left(u_{n}\right) d t \rightarrow+\infty, \quad \forall u_{n} \rightarrow u \in \partial \Lambda_{0}
$$

(1) If $u \equiv 0$, then

$$
\left|u_{n}\right|_{\infty} \rightarrow 0, \quad n \rightarrow+\infty
$$

Then we have

$$
f\left(u_{n}\right) \geq 6\left|u_{n}\right|_{\infty}^{2-\beta} \rightarrow+\infty, \quad n \rightarrow+\infty
$$

So in this case we have

$$
\liminf f\left(u_{n}\right)=+\infty \geq f(u)
$$

(2) If $u \neq 0$. By the weakly lower semi-continuity for norm, we have

$$
\liminf \left\|u_{n}\right\| \geq\|u\|>0
$$

So by Gordon's lemma, we have

$$
\begin{aligned}
\liminf f\left(u_{n}\right) & =\liminf \left(\frac{1}{2} \int_{0}^{1}\left|\dot{u}_{n}\right|^{2} d t\right) \int_{0}^{1}\left(h-V\left(u_{n}\right)\right) d t=+\infty \\
& \geq \frac{1}{2} \int_{0}^{1}|\dot{u}|^{2} d t \int_{0}^{1}(h-V(u)) d t=f(u) .
\end{aligned}
$$

Lemma 3.3 $\bar{\Lambda}_{0}$ is a weakly closed subset of $H^{1}$.

Proof By Sobolev's embedding theorems, the proof is obvious.

Lemma 3.4 The functional $f(u)$ is coercive on $\Lambda_{0}$.

Proof By the definition of $f(u)$ and the assumption (V2), we have

$$
f(u)=\frac{1}{2} \int_{0}^{1}|\dot{u}|^{2} d t \int_{0}^{1}(h-V(u)) d t \geq \frac{h}{2} \int_{0}^{1}|\dot{u}|^{2} d t, \quad \forall u \in \Lambda_{0} .
$$


Lemma 3.5 The functional $f(u)$ attains the infimum on $\Lambda_{0}$; furthermore, the minimizer is non-constant.

Proof By Lemma 2.2 and Lemmas 3.1-3.3, we know that the functional $f(u)$ attains the infimum in $\Lambda_{0}$; furthermore, we claim that

$$
\inf _{\Lambda_{0}} f(u)>0
$$

since otherwise, $u_{0}(t)=$ const attains the infimum 0 , then by the symmetry of $\Lambda_{0}$, we have $u_{0}(t) \equiv 0$, which contradicts the definition of $\Lambda_{0}$. Now we know that the minimizer is non-constant.

\section{Competing interests}

The authors declare that they have no competing interests.

\section{Authors' contributions}

XW proved the main theorem, SS participated in the proof and helped to draft the manuscript. Both authors read and approved the final manuscript.

\section{Author details}

'Department of Mathematics, Yibin University, Yibin, Sichuan 644007, China. ²Department of Mathematics, Sichuan University, Chengdu, Sichuan 610064, China.

\section{Acknowledgements}

This study was supported by the Scientific Research Fund of Sichuan Provincial Education Department (11ZA172) and the Scientific Research Fund of Science Technology Department of Sichuan Province (2011 JYZ010).

Received: 14 November 2013 Accepted: 10 February 2014 Published: 19 Feb 2014

\section{References}

1. Ambrosetti, A, Coti Zelati, V: Closed orbits of fixed energy for singular Hamiltonian systems. Arch. Ration. Mech. Anal. $112,339-362(1990)$

2. Ambrosetti, A, Coti Zelati, V: Periodic Solutions for Singular Lagrangian Systems. Springer, Berlin (1993)

3. Carminati, $\mathrm{C}$, Sere, E, Tanaka, $\mathrm{K}$ : The fixed energy problem for a class of nonconvex singular Hamiltonian systems. J. Differ. Equ. 230, 362-377 (2006)

4. Tanaka, K: Periodic solutions for singular Hamiltonian systems and closed geodesics on non-compact Riemannian manifolds. Ann. Inst. Henri Poincaré, Anal. Non Linéaire 17, 1-33 (2000)

5. Pisani, L: Periodic solutions with prescribed energy for singular conservative systems involving strong forces. Nonlinear Anal. TMA 21, 167-179 (1993)

6. Gordon, WB: Conservative dynamical systems involving strong forces. Trans. Am. Math. Soc. 204, 113-135 (1975)

10.1186/1687-2770-2014-42

Cite this article as: Wang and Shen: New periodic solutions for a class of singular Hamiltonian systems. Boundary Value Problems 2014, 2014:42

\section{Submit your manuscript to a SpringerOpen ${ }^{\circ}$ journal and benefit from:}

- Convenient online submission

- Rigorous peer review

- Immediate publication on acceptance

Open access: articles freely available online

- High visibility within the field

- Retaining the copyright to your article 